\title{
Allopathic residencies: Current and former residents' viewpoints
}

\section{To the Editor:}

Dr Pecora's article "Factors influencing osteopathic physicians' decisions to enroll in allopathic residency programs" (JAOA 1990;90:527-533) was interesting.

I am currently enrolled in an osteopathic general practice residency. I did so to repay a debt to the osteopathic profession for allowing me to learn the art of medicine. Nonetheless, this indebtedness does not mean that I am completely satisfied with the situation. The educators earn less money than the program administrators at this hospital as well as other training hospitals. If salaries were higher for teachers in osteopathic postgraduate programs, and if such programs were willing to employ allopathictrained DOs, we as a profession would prove our dedication to the education of our future residents.

I believe that many of my contemporaries enrolled in allopathic programs to receive a better education. Dr Pecora's statement in his conclusion, "Many hospitals need house officers and would probably welcome such a solution to their personnel shortages," is a sad commentary on the osteopathic medical profession's view of residents. We residents are enrolled in these programs to learn-not to do "scut work."

MICHAEL BARON, DO

Second-Year Resident

Department of General Practice

Northlake Regional Medical Center

Tucker, Ga

\section{To the Editor:}

As an osteopathic physician who trained in an allopathic residency program, I read Dr Pecora's article with great interest. It is refreshing and encouraging to see a respected teacher within our profession ask - and attempt to answer-these difficult questions. It certainly is hard to accept the fact that many osteopathic residency positions may be just that-simply positions created to fill a void or a need for house officers to supply a service. As such, comprehensive leadership and faculty are lacking that would assure broad-based training necessary to produce highly qualified physicians.

Because I recently completed an allopathic training program in psychiatry, I can certainly identify with the pros and cons Dr Pecora addressed in his article. I am also aware that allopathic residency programs, by no means, neither have all the answers, nor can they produce highquality physicians without raw quality "material." Osteopathic medical graduates are that highquality raw "material."

Certainly, physicians who trained in osteopathic programs have a valid concern about letting those physicians who trained in allopathic programs "return to the fold." Nevertheless, we as a profession must rise above these individual concerns to keep osteopathic medicine strong. Like most of my allopathically trained colleagues, I want to maintain our osteopathic heritage, not abandon it.

Unfortunately, it seems at times that the profession would prefer to abandon its members. Those physicians who feel alienated may have valid gripes. They may also have personal issues that they need to set aside to keep the profession strong. Individuals who are unable to set these personal issues aside probably do not participate in any professional groups anyway. The majority of DOs should not be punished because of the behavior of the minority of osteopathic physicians.

Examining these issues is healthy for our profession. I hope that this dialogue continues vigorously with the goal of maintaining the profession's strength. Attaining this goal may mean we all need to sacrifice a little of ourselves. We can start by examining the needs of our graduates and the necessary improvements needed to be made in our training programs so that the whole question of allopathic residency programs becomes moot.

STEVEN J. KARP, DO
Department of Psychiatry
and Behavioral Medicine
Geisinger Medical Center
Danville, Pa

\section{Response}

\section{To the Editor:}

I am not sure I understand what Dr Baron means by enrolling in an osteopathic residency "to repay a debt to the osteopathic profession for allowing me to learn the art of medicine." If he is suggesting that he remained in an osteopathic program out of loy(continued on page 752) 


\section{In hypertension, There's safety in these numbers.}
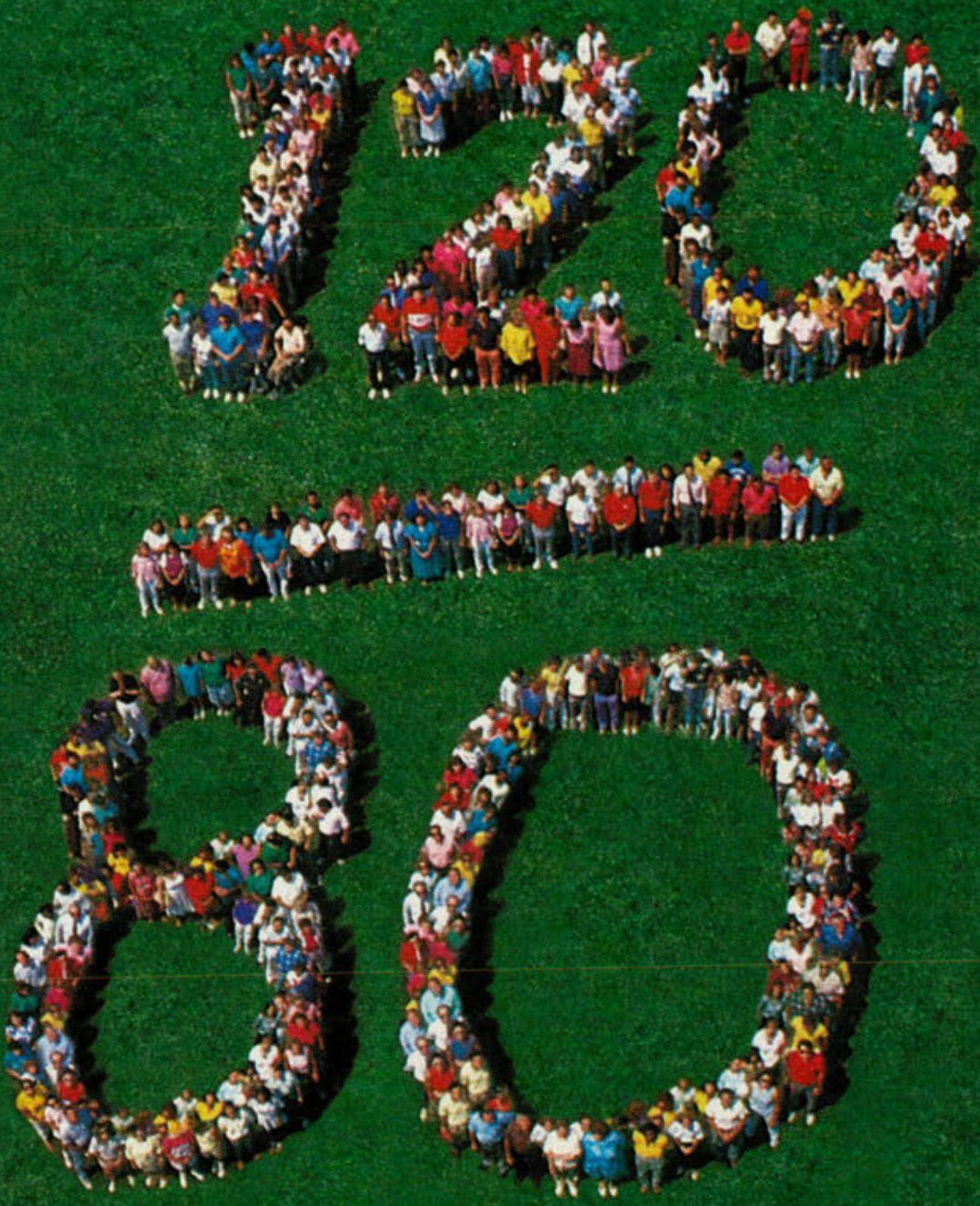

\section{MINIPRESS (prazosin $\mathrm{HCl}$ ) Blood pressure control that leaves other CHD risk factors unaffected}

1. Effectively reduces high blood pressure

2. Does not adversely affect the lipid profile $e^{2 \cdot 6}$

3. Does not impair exercise capacity'

4. Has no significant effect on glucose metabolism ${ }^{7.8}$

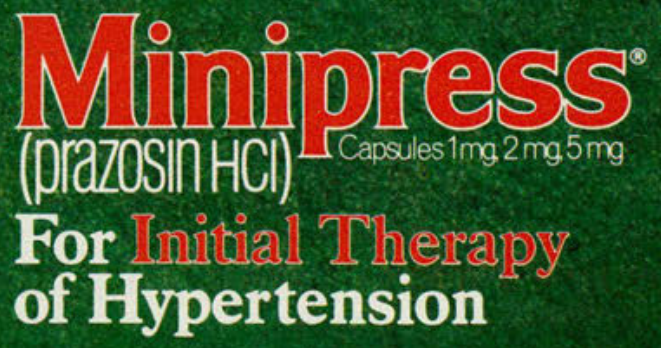

Most common side effects, generally mild and transient, are: dizziness, headache, drowsiness, palpitations, and nausea. Syncope has been reported in about $0.15 \%$ of patients at the recommended initial dose of $1 \mathrm{mg}$. 
References: 1. Lund-Johansen P: Hemodynamic changes at rest and during exercise in long-term prazosin therapy for essential hypertension, in Prazosin Clinical Symposium Proceedings. Published as a special report by Postgraduate Medicine. New York, McGraw.
Hill Co, 1975, pp 45-52. 2. Komajda M. Prazosin and lipids: A study of the effects of prazosin Hili Co, 1975, pp 45-52.2. Komajda M: Prazosin and lipids: A study of the eftects of prazzosien
on blood lipids in hypertensive patients. Impact int December 1986; (speciat issue): 1-4 3. Rouffy J, Jaillard J: Effects of two antihypertensive agents on lipids, lipoproteins, an apoproteins A and B. Comparison of prazosin and atenolol, Am J Med 1986,80(supp

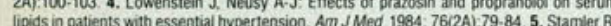
Stamler $J$ Gosch $F C$, et at: Initial antihypertensive drug therapy. A comparison of alph blocker (prazosin) and diuretic (hydrochlorothiazide): Final report of a randomized, con trolled trial. In press. 6. Leren P. Helgeland A, Holme I, et al: Effect of propranolol and prazosin on blood lipids: The Osio Study. Lancet 1980; II:4-6. 7. Kwan CM. Shepherd AMM. Johnson J. et al: Forearm and finger hemodynamics, blood pressure control and lipid changes in patients with diabetic hypertension treated with atenolol and prazosin. Clin
Pharmacol Ther 1988,44:202-209. 8. Leichter SB. Baumgardner B: Eftects of chronic prazosin therapy on intermediary metabolism in diabetic patients. $J$ Cardiovasc Med 1981.(special suppl): $38-42$

Briel Summary

For Oral Use

NOICATIONS AND USAGE: MINIPRESS (prazosin hydrochloride) is indicated in the treat ment of hypertension. It is mild to moderate in activity and can be used as the initial agent on in a general treatment program in conjunction with a diuretic and/or other antihypertensive
drugs as needed. CONTRAINDICATIONS: None known. WARNINGS: MINIPRESS may cause syncope with sudden loss of consciousness. In most cases this is believed to be due to an excessive postural hypotensive effect, although occasionally the syncopa episode has been preceded by a bout of severe tachycardia with heart rates of $120-16 \mathrm{C}$ beats per minute. Syncopal episodes have usually occurred within 30 to 90 minutes of the initial dose of the drug: occasionally they have been reported in association with rapic dosage increases or the introduction of another antihypertensive drug into the regimen 0 approximately $1 \%$ in patients given an initial dose of $2 \mathrm{mg}$ or greater. Clinical trials conducted during the investigational phase of this drug suggest that syncopal episodes conducted during the investigational phase of this drug suggest that syncopal episodes
can be minimized by limiting the initial dose of the drug to $1 \mathrm{mg}$. by subsequently increasing the dosage slowly, and by introducing any additional antiliypertensive drug: into the patient's regimen with caution (see DOSAGE AND ADMINISTRATION). Hypoten sion may develop in patients given MINIPRESS who are also receiving a beta-blocke such as propranolol. If syncope occurs, the patient should be placed in the recumbent position and treated supportively as necessary. This adverse effect is sell-limiting and in most cases does not recur after the initial period of therapy or during subsequent dose titration. The patient should also be cautioned to avoid situations where injury could resull Should syncope occur during the initiation of MINIPRESS therapy. PRECAUTIONS: Informa tion for Patients: Diziness or drowsiness may occur after the first dose of this medicine Avoid driving or performing hazardous tasks for the first 24 hours after taking this medicine or when the dose is increased. Dizziness, lightheadedness or tainting may occur, especially Then fising from a lying or sitting position. Getting up slowly may help lessen the problem if the weather is hot. While taking MINIPRESS, be careful in the amount of alcohol you drink Also, use extra care during exercise or hot weather, or it standing for long periods. Check with your physician if you have any questions. Drug Interactions: MiNIPRESS has been administered without any adverse drug interaction in limited clinical experience to date with the following: (1) cardiac glycosides-digitalis and digoxin; (2) hypoglycemics-insulin, chiorpropamide. phenformin, tolazamide, and tolbutamide; (3) tranquilizers and sedatives-

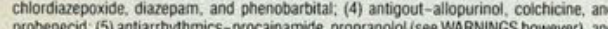
probenecid, (5) antiarthythmics-procainamide, propranolor (see WARNINGS however), and indomethacin, and phenylbutazone. Addition of a diuretic or other antihypertensive, aspenin MINIPRESS has been shown to cause an additive hivpotensive effect. Orug/Laboratory Jes Interactions: False positive results may occur in screening tests for pheochromocytoma in
patients who are being treated with prazosin. If an levated VMA is found, prazosin should be discontinued and the patient retested after a month. Laboratory Tests: In clinical studies in which lipid profiles were followed, there were generally no adverse changes noted between pre-and post-treatment lipid levels. Carcinogenesis, Mutagenesis, Impairment of Fer. tility: No carcinogenic potential was demonstrated in an 18 month study in rats with MINIPRESS (prazosin hydrochloride) at dose levels more than 225 times the usual maximum recommended human dose of $20 \mathrm{mg}$ per day. MINIPRESS was not mutagenic in in vivo genetic toxicology studies. In a fertility and general reproductive performance study in rats both males and temales, treated with $75 \mathrm{mg} / \mathrm{kg}$ (225 times the usual maximum recom-
mended human dose), demonstrated decreased fertility while those treated with $25 \mathrm{mg} / \mathrm{kg}$ 75 times the usual maximum recommended human dose) did not. In chronic studies year or more) of MINIPRESS in rats and dogs, testiculas changes consisting of atrophy an necrosis occurred at $25 \mathrm{mg} / \mathrm{kg} / \mathrm{day}$ (75 times the usual maximum recommended hum an dose). No testicular changes were seen in rats or dogs at $10 \mathrm{mg} / \mathrm{kg} / \mathrm{day}$ ( 30 times the usua maximum recommended human dose). In view of the testicular changes observed in animals, 105 patients on long term MINIPRESS therapy were monitored for 17-ketosteroid excretion and no changes indicating a drug effect were observed. In addition, 27 males on MINIPRESS tor up to 51 months did not have changes in sperm morphology suggestive o drug effect. Usage in Pregnancy: Pregnancy Category C. There are no adequate and weil controlled studies which establish the safety of MiviPRESS (prazosin $\mathrm{HC}$ ) in pregnant The potential risk to she mould be used during pregnancy only if the potential benefit justifie: be excreted in small amounts in human milk. Caution should be exercised when MiNiPPESS is administered to a nursing win human milk. Caution should be exercised when MINIPARESS have not been established. ADVFRSE REACTIONS: Clinical trials were conducted on more than 900 patients. During these trials and subsequent marketing experience, the most trequent reactions associated with MINIPRESS therapy are: dizziness $10.3 \%$, headache
7.896 , drowsiness $7.69 \%$, lack of energy $6.9 \%$, weakness $6.5 \%$, palpitations $5.39 \%$, and 7.8\% . drowsiness $7.69 \%$, lack of energy $6.99 \%$, weakness $6.5 \%$, palpitations $5.3 \%$, and nausea $4.9 \%$. In most instances side effects have disappeared with continued therapy or have been tolerated with no decrease in dose of drug. Less frequent adverse reactions which are reported to occur in 1-4\% of patients are: Gastrointestinal: vomiting, diarnhea, constipa tion, Cardiovascular: edema, orthostatic hypotension, dyspnea, syncope; Central Nervous
System, vertigo, depression, nervousness; Dermatologic: rash; Genitourinary: urinary freSystem: vertigo, depression, nervousness; Dermatologic: rash; Genitourinary urinary fre-
quency. EENT. blurred vision, reddened sclera, epistaxis, dry mouth, nasal congestion. In addition, fewer than $1 \%$ of patients have reported the foliowing (in some instances, exact causal relationships have not been established): Gastrointestinat: abdominal discomfor and/or pain, liver function abnormalities, pancreatitis; Cardiovascular tachycardia; Central planus, Genitourinary incontinence, impotence, priapism, EENT: tinnitus; Other: diaphoresis, fever. Single reports of pigmentary mottling and serous retinopathy. and a few reports cataract development or disappearance have been reported. OVERDOSAGE: Should overdosage lead to hypotension, support of the cardiovascular system is of first importance.
Restoration of blood pressure and normalization of heart rate may be accomplished by Restoration of blood pressure and normalization of heart rate may be accomplished by
keeping the patient in the supine position. If this measure is inadequate, shock should first be keeping the patient in the supine position. If this measure is inadequate, shock should firstbe
treated with volume expanders. If necessary, vasopressors should then be used. Renal function should be monitored and supported as needed. Laboratory data indicate The dose of MINIPRESS should be adjusted according to individual blood pressure response. Initial Dose: $1 \mathrm{mg}$ two or three times a day. Maintenance Dose: Dosage may
resure response. Initial Dose: $1 \mathrm{mg}$ two or three times a day. Maintenance Dose: Dosage may
be stowly increased to a total daily dose of $20 \mathrm{mg}$ given in divided doses. The therapeutic dosages most commonly employed have ranged from $6 \mathrm{mg}$ to $15 \mathrm{mg}$ daily given in divided doses. Doses higher than $20 \mathrm{mg}$ usually do not increase efficacy: however a few patients may benefit from further increases up to a daily dose of $40 \mathrm{mg}$ given in divided
doses. After initial titration some patients can be maintained adequately on a twice daily dosage regimen. Use With Other Drugs: When adding a diuretic or other antihypertensive agent, the dose of MiNIPRESS should be reduced to $1 \mathrm{mg}$ or $2 \mathrm{mg}$ three times a day and retitration then carried out. Hevised December 1988

alty, he should be commended. If he is not totally satisfied with his situation, then perhaps Dr Baron should reevaluate his goals.

Clearly, Dr Baron misunderstood one of my concluding statements, "Many hospitals need house officers and would probably welcome such a solution to their personnel shortages." I was not suggesting that osteopathic residents, or any other residents for that matter, be put in any institution simply as warm bodies to do "scut work." What I meant to convey was that hospitals without training programs would be very willing, I believe, to fund training programs. Such financial support would enable the osteopathic profession to institute highly, qualified academic training programs in larger hospitals with a significant number of diverse patients. Having been a resident once myself, I certainly do not advocate the "service residency" type of program. Likewise, residents are not around to do "scut work."

Concerning Dr Karp's kind comments, I would like to thank and commend him for his suggestion that the profession must rise above individual concerns to maintain the strength of osteopathic medicine. In my article, I suggested that a great deal of time and effort must be spent in evaluating the problems related to our residency programs. It may take 5 or 10 years before we can approach these problems with an intelligent plan. In order to do this, I believe that the profession, including academically qualified individuals, must come together under the auspices of the American Osteopathic Asso- ciation and work toward a solution.

One thing is certain: Many of our residency programs are strictly service-oriented. We must dispense with this type of training program and introduce very structured, educational, and exciting programs for our residents.

ANDREW A. PECORA, DO Professor and Chairman Department of Medicine University of Medicine and Dentistry of New Jersey -School of Osteopathic Medicine

Stratford, NJ

\section{Commenting on L- tryptophan and osteopathic physicians in allopathic residencies}

\section{To the Editor:}

In response to Dr Northup's editorial "Natural is not always healthy" (JAOA 1990;90:505), it should have been obvious early on that something other than Ltryptophan was causing eosinophilia-myalgia syndrome (EMS). The Centers for Disease Control has traced this EMS outbreak to one foreign manufacturer of Ltryptophan, which implies contamination.

I do not see such an outcry over drugs used in medicine that often cause deleterious effects. For example, chemotherapeutic agents are known to produce additional malignancies in as many as $25 \%$ of the patients receiving such treatment. Natural may not always be healthy, as Dr (continued on page 759) 